\title{
MicroRNA-34b-5p inhibits proliferation, stemness, migration and invasion of retinoblastoma cells via Notch signaling
}

\author{
SHURONG ZHANG and ZHE CUI \\ Department of Ophthalmology, The Third Affiliated Hospital of Qiqihar Medical University, \\ Qiqihar, Heilongjiang 161000, P.R. China
}

Received May 15, 2019; Accepted October 20, 2020

DOI: $10.3892 /$ etm.2021.9686

\begin{abstract}
Retinoblastoma (RB) is one of the most common forms of childhood intraocular cancer. While the occurrence of $\mathrm{RB}$ is traditionally associated with dysregulation of the $R B 1$ gene, efforts have been made to assess the role of several other pathways that may result in RB. The Notch signaling pathway has been identified as one of the sentinel pathways in retinal development and has been indicated to serve as a tumor suppressor. However, epigenetic modifications of the Notch signaling pathway, and their consequences on tumor establishment and progression, have received little attention. The present study attempted to elucidate the microRNA (miR)-mediated dysregulation of the Notch signaling pathway and its implications on tumor initiation. Upon recruitment of patients with $\mathrm{RB}$ (age, 4-25 months), the levels of miR-34b-5p were determined in tumor and adjacent healthy tissues. Simultaneously, the serum levels of miR-34b-5p were measured in tumor and healthy samples using reverse transcriptase-quantitative PCR (RT-qPCR). Binding of miR-34b-5p to Notch1 and Notch2 were confirmed bioinformatically. In vitro studies were performed in Y79 and Weri-Rb-1 RB cell lines. The cell lines were transfected with miR-34b-5p constructs and miR-34b-5p overexpression was confirmed using RT-qPCR. The impact of miR-34b-5p overexpression on cell growth and cancer stemness markers (Sox-2, Nanog, and CD133) was examined. The expression levels of Notch1 and Notch2 were evaluated in the presence of miR-34b-5p. The rescue of cell growth and cancer stemness phenotypes was evaluated by co-transfection of miR-34b-5p with Notch1 or Notch2. The results of the present study indicated that the expression levels of miR-34b-5p were reduced in patient tissues and serum samples compared with those in healthy tissues and samples. Notch1 and Notch2 expression
\end{abstract}

Correspondence to: Professor Zhe Cui, Department of Ophthalmology, The Third Affiliated Hospital of Qiqihar Medical University, 27 Taishun Street, Qiqihar, Heilongjiang 161000, P.R. China E-mail: cz665008@126.com

Key words: microRNA-34b-5p, retinoblastoma, proliferation, stemness, Notch1, Notch2 level was negatively correlated with the expression level of miR-34b-5p. Overexpression of miR-34b-5p resulted in reduced cell proliferation, migration, invasion and cancer stemness compared with the control group. Further in vivo experiments confirmed the inhibitory effects of miR-34b-5p on $\mathrm{RB}$ cell proliferation. Upon co-transfection of miR-34b-5p with Notch1 or Notch2, these phenotypes were rescued with reversal of cell growth and tumor sphere formation. Collectively, the results indicated that miR-34b-5p functions as a tumor suppressor in RB via regulating the Notch signaling pathway. Therefore, miR-34b-5p may be explored for its utility as a therapeutic target in RB.

\section{Introduction}

One of the most common causes of childhood intraocular cancer is retinoblastoma (RB) (1). The incidence of RB is constant worldwide with 1 case per 15,000-20,000 live births, equating to $\sim 9,000$ new cases per year (2). However, the greatest burden of RB is observed in Asia and Africa, which are regions with higher birth rate compared with Europe, USA or Canada (1). The regions with higher prevalence of RB have also been associated with higher mortality, which may be attributed to delayed diagnosis due to the lack of familial awareness, access to healthcare and the poor socioeconomic status (1).

$\mathrm{RB}$ is usually initiated by a random mutation in the $R B 1$ gene of the photoreceptor cells of the retina (2). The study of the $R B 1$ gene, which was the first tumor suppressor gene to be identified, gave rise to the famous two-hit hypothesis for cancer development propounded by Knudson et al (3). The multifunctional protein $\mathrm{pRb}$ (the product of $R B 1$ ) has been associated with several tumor suppressive functions (4). Additionally, its role has also been investigated in the maintenance of genome stability and other epigenetic modifications (5), for example, decreases in pRB chromatin are associated with increases in spontaneous $\gamma \mathrm{H} 2 \mathrm{AX}$ deposition and aneuploidy (5).

However, the accuracy of the two-hit hypothesis was challenged with the genomic revolution, questioning the simplicity and reliability of RB development on the two-hit hypothesis alone without taking into consideration chromosomal and epigenetic alterations (6). This highlighted the requirement for further investigation in order to understand the initiation and progression of $\mathrm{RB}$. 
Studies in humans and mice have indicated that biallelic inactivation of $R B 1$ resulted in increased copy numbers of $M Y C N, E 2 F 3, D E K, K L F 14$ and MDM4 as well as decreased tumor suppressor genes $C D H 11$ and NFGR (7-9). These additional alterations were reported to be necessary for the development and progression of RB (10). While the role of genetics has been investigated, the role of epigenetic modifications in the molecular mechanisms of RB remains relatively unexplored (11).

In the current study, the epigenetic modifications of Notch signaling in RB were investigated, as Notch receptors serve an important role in the specification and survival of stem and progenitor cells in retinal development (12). Furthermore, it has been indicated that Notch1 interacts with $\mathrm{pRb}$ via intracellular domains, resulting in $\mathrm{pRb}$ inactivation, which is therefore likely to contribute to the oncogenic activity of Notch1 (13). In addition, the Notch signaling pathway has been associated with increased cell proliferation, angiogenesis, recurrence and maintenance of cancer stem cell population and chemoresistance in several cancer types, such as hepatocellular carcinoma, breast cancer and lung cancer (14-18).

Dysregulation of the Notch signaling pathway either due to overexpression of the ligand or aberrant receptor expression has been reported in several solid tumor studies, such as thyroid cancer, lung cancer and intracranial tumors (19). Moreover, activation of the Notch1 gene signature independently of common mutations has also been reported in chronic lymphocytic leukemia (20). While targeting the Notch pathway has been proposed as a therapeutic alternative in RB, a deeper understanding of the epigenetic regulatory mechanisms of Notch signaling in RB remains elusive (18).

The present study is amongst the first studies, to the best of our knowledge, to explore microRNA (miR)-mediated epigenetic modulation of the Notch signaling pathway and its influence on phenotypes of tumor progression in RB. A previous study identified a 30-miR core, which was revealed to be upregulated in RB in 12 patient tissues, using high-throughput microarray analysis (11). These $30 \mathrm{miRs}$ have been reported to target 182 cancer-associated genes, albeit none of them was indicated to target the Notch genes. However, via a literature search, miR-34b-5p was revealed to be associated with Notch dysregulation in thyroid carcinoma and uterine cervix adenocarcinoma $(21,22)$. As the effect of miR-34b-5p-mediated Notch dysregulation in RB development has not been yet investigated, the current study was designed to address this question. It was hypothesized that miR-34b-5p-regulated Notch signaling may drive tumor progression in RB.

\section{Materials and methods}

Patient samples and inclusion criteria. The current study was performed at The Third Affiliated Hospital of Qiqihar Medical University (Qiqihar, China) in accordance with the principles of the Declaration of Helsinki and was approved by the clinical research Ethics Committee of The Third Affiliated Hospital of Qiqihar Medical University (Qiqihar, China). Patients $(n=10)$ aged 4-25 months diagnosed with RB and undergoing opthalmectomy between January 2014 and January 2017 at the hospital, with no preoperative chemo- and radiotherapy, were recruited in the present study and provided written informed consent. The diagnosis of retinoblastoma was based on the WHO criteria (23). To avoid RB contamination, healthy tissue was resected separately from an opposite quadrant of the tumor tissue ( $>5 \mathrm{~mm}$ from the tumor margin). Patient sera were also obtained. Briefly, the whole blood was allowed to clot by leaving it undisturbed at room temperature for 15-30 $\mathrm{min}$. The clot was removed by centrifuging at 1,000-2,000 $\mathrm{xg}$ for $10 \mathrm{~min}$ and the supernatant serum was collected. Simultaneously, age-matched normal sera were also collected from outpatients of physical examination excluding patients with infections, tumors and various congenital diseases. All sera were immediately snap frozen in liquid nitrogen and stored at $-80^{\circ} \mathrm{C}$ for further use. The clinical characteristics of the patients with $\mathrm{RB}$ and normal participants are listed in Table S1.

Cell line maintenance and transfection. Two human-derived RB cell lines, Weri-Rb-1 and Y79, were obtained from the American Type Culture Collection and maintained as per the repository's instructions. Briefly, the cells were seed in 24-well plate and cultured in RPMI-1640 medium (Gibco; Thermo Fisher Scientific, Inc.) containing 10\% FBS (Gibco; Thermo Fisher Scientific, Inc.) and antibiotics (penicillin and streptomycin; $100 \mathrm{U} / \mathrm{ml} \mathrm{each)}$ at $37^{\circ} \mathrm{C}$ in a $5 \% \mathrm{CO}_{2}$ incubator till $80 \%$ confluence. Plasmids (400 ng) containing genes for Notch1 or Notch2 in pcDNA3.1 vectors (Shanghai GenePharma Co., Ltd.) and $0.14 \mu \mathrm{l} 20 \mu \mathrm{M}$ stock of miR-34b-5p (5'-AGGCAG UGUAAUUAGCUGAUUGU-3') or control miR (5'-UUCUCC GAACGUGUCACGUTT-3') in $30 \mu 1$ medium without serum or antibiotics were mixed with $0.75 \mu$ l Lipofectamine ${ }^{\circledR} 2,000$ (Invitrogen; Thermo Fisher Scientific, Inc.) for $15 \mathrm{~min}$ at room temperature to form transfection complexes. The complexes were added into each single well of 24-well plate cultured RB cells. Assays were performed $48 \mathrm{~h}$ post-transfection.

Reverse transcription-quantitative PCR (RT-qPCR). As per the manufacturer's protocol, total RNA was extracted using the TRIzol ${ }^{\circledR}$ reagent (Invitrogen; Thermo Fisher Scientific, Inc.) from RB cells (Y79 and Weri-Rb-1) and tissue samples. The RNA was converted to cDNA using a TaqMan ${ }^{\circledR}$ MicroRNA Reverse Transcription kit (Applied Biosystems; Thermo Fisher Scientific, Inc.). The thermocycling conditions were as follows: $16^{\circ} \mathrm{C}$ for $30 \mathrm{~min}, 42^{\circ} \mathrm{C}$ for $30 \mathrm{~min}$ and $85^{\circ} \mathrm{C}$ for 5 min. RT-qPCR was performed using a TaqMan ${ }^{\circledR}$ Universal PCR Master Mix II (Applied Biosystems; Thermo Fisher Scientific, Inc.) with small nuclear RNA U6 as the endogenous control. The RT-qPCR of mRNA was performed with Superscript III Platinum SYBR Green One-Step qRT-PCR kit (Invitrogen; Thermo Fisher Scientific, Inc.) using $\beta$-actin as the house-keeping gene. The RT-qPCR were run on an ABI 7500 Real-Time PCR system (Applied Biosystems) at $95^{\circ} \mathrm{C}$ for $2 \mathrm{~min}$, followed by 30 cycles of amplification at $94^{\circ} \mathrm{C}$ for $40 \mathrm{sec}$, annealing at $55^{\circ} \mathrm{C}$ for $40 \mathrm{sec}$ and extension at $72^{\circ} \mathrm{C}$ for $1 \mathrm{~min}$, with a final elongation step at $72^{\circ} \mathrm{C}$ for $10 \mathrm{~min}$, and the relative gene expression was estimated using the $2^{-\Delta \Delta \mathrm{cq}}$ method (24). The primer sequences used for the gene expression analysis were as follows: Notch1 forward, 5'-GGGCTA ACAAAGATATGCAG-3' and reverse, 5'-ACTGAACCT GACCGTACAGTTGGCAAAGTGGTCCAG-3'; Notch2 forward, 5'-AATCCCTGACTCCAGAACG-3' and reverse, 5'-TGGTAGACCAAGTCTGTGATGAT-3'; CD133 forward, 
5'-GAAAAGTTGCTCTGCGAACC-3' and reverse, 5'-CTC GACCTCTTTTGCAATCC3'; SOX-2 forward, 5'-GGGAAA TGGAGGGGTGCAAAAGAGG-3' and reverse, 5'-TTGCGT GAGTGTGGATGGGATTGGTG-3'; Nanog forward, 5'-TCC TCCTCTTCCTCTATACTAAC-3' and reverse, 5'-CCCACA ATCACAGGCATAG-3'; and $\beta$-actin forward 5'-AAGGGA CTTCCTGTAACAATGCA-3' and reverse, 5'-CTGGAA CGGTGAAGGTGACA-3', miR-34b-5p forward 5'-GTCGTA TCCAGTGCAGGGTCCGAGGTATTCGCACTGGATACG ACCAATCA-3' and reverse, 5'-GCCTAGGCAGTGTCATTA GC-3'; and U6 forward 5'-CTCGCTTCGGCAGCACA-3' and reverse, 5'-AACGCTTCACGAATTTGCGT-3'.

MTT assay. RB cell (Weri-Rb-1 and Y79) proliferation was assessed using an MTT assay (Beyotime Institute of Biotechnology). Briefly, for each cell line, 5,000 cells were seeded in a 96-well plate. MTT was added to each well on day $0,1,2,3$ and 4 post-transfection at a final concentration of $0.5 \mathrm{mg} / \mathrm{ml}$, and incubated for $4 \mathrm{~h}$ at $37^{\circ} \mathrm{C}$. The formation of formazan crystals was assessed under the microscope and the crystals were dissolved using DMSO. The absorbance was measured in a microplate reader at $450 \mathrm{~nm}$.

Cell counting kit-8 (CCK-8) assay. Cellular proliferation was also measured using a commercial CCK-8 assay (Beyotime Institute of Biotechnology). A total of 5,000 cells of the respective RB cell lines were seeded in a 96-well plate. Following transfection (day 0, 1, 2, 3 and 4), CCK reagent was added to each well at a final dilution of 1:10. The cells were subsequently incubated at $37^{\circ} \mathrm{C}$ for $2 \mathrm{~h}$ and the absorbance was measured at $450 \mathrm{~nm}$ in a microplate reader.

Western blotting. The transfected cells (Y79 and Weri-Rb-1 cells) were harvested using a commercial RIPA lysis buffer (Beyotime Institute of Biotechnology) and total protein content was estimated using a BCA assay (Beyotime Institute of Biotechnology). A total of $20 \mu \mathrm{g}$ total protein/lane was separated using 10\% SDS-PAGE and electroblotted to a nitrocellulose membrane (Bio-Rad Laboratories, Inc.). Following transfer, the membranes were blocked for $1 \mathrm{~h}$ at room temperature with $5 \%$ non-fat dried milk. The excess buffer was removed by washing in PBS buffer and the membranes were incubated with primary antibodies including Notch1 (cat. no. sc-376403; 1:1,000), Notch2 (cat. no. sc-518049; 1:500), CD133 (cat. no. sc-19365; 1:500), SOX-2 (cat. no. sc-365823; 1:500), Nanog (cat. no. sc-374103; $1: 500)$ and $\beta$-actin (cat. no. sc-47778; 1:1,000). overnight at $4^{\circ} \mathrm{C}$. After washing using TBST (Tris Buffered Saline with $0.1 \%$ Tween 20 ), the membranes were incubated for $1 \mathrm{~h}$ with HRP-conjugated species-specific secondary antibodies (horseradish peroxidase conjugated goat anti-rabbit Immunoglobulin G, cat. no. sc-2004; and horseradish peroxidase conjugated goat anti-mouse Immunoglobulin G, cat. no. sc-2005; each at 1:10,000) at room temperature. All antibodies were purchased from Santa Cruz Biotechnology, Inc. Subsequently, the blots were developed using an ECL kit (Beyotime Institute of Biotechnology). The blots were developed on an X-ray film (Kodak) and densitometry analysis was performed using ImageJ software (version 1.48; National Institutes of Health).
Tumor sphere formation assay. For this assay, 24-well plates with an ultra-low attachment surface (Corning Inc.) were used. Transfected and non-transfected Y79 and Weri-Rb-1 cells were seeded at a density of 5,000 cells/well. All cells were cultured in RPMI-1640 medium without serum, but supplemented with $20 \mathrm{ng} / \mathrm{ml}$ epidermal growth factor and $10 \mathrm{ng} / \mathrm{ml}$ basic fibroblast growth factor (both from Invitrogen; Thermo Fisher Scientific, Inc.). The cells were maintained for 7 days at $37^{\circ} \mathrm{C}$ in a $5 \% \mathrm{CO}_{2}$ incubator. Using a light microscope (Nikon Corporation; magnification, x200) tumor spheres were imaged and only spheres with diameter over $50 \mu \mathrm{m}$ were counted using imageJ software (version 1.48; National Institutes of Health).

Xenograft tumor model. A total of $10 \mathrm{BALB} / \mathrm{c}$ nude mice (male, 4-week-old, 15-20 g; Charles River Laboratories, Inc.) were randomly divided into two groups. One group was subcutaneously injected with $1 \times 10^{7}$ Y79 cells with miR-34b-5p overexpression, and the other group was injected with cells transfected with control miR. All mice were injected subcutaneously on their right flank. During the experimental phase, all mice had free access to food and water. To minimize any suffering of the animals, anesthetics and analgesics were used for all surgical experiments. Animals were anesthetized with an intraperitoneal injection of $40 \mathrm{mg} / \mathrm{kg}$ sodium pentobarbital. When lack of movement and absence of corneal reflex, but presence of heartbeat and respiration were observed, the surgical experiments were performed. All mice were given Meloxicam $(2.5 \mathrm{mg} / \mathrm{kg}$, s.c.) every $12 \mathrm{~h}$ for three days after surgery. The mice were maintained in an atmosphere of $60 \%$ humidity at a temperature of $26-28^{\circ} \mathrm{C}$ with a uniform dark-light cycle of $12 \mathrm{~h}$ each of light and darkness. After 24 days, all mice were sacrificed by $\mathrm{CO}_{2}$ inhalation $(20 \%$ of the cage $\mathrm{vol} / \mathrm{min}$ ) followed by cervical dislocation. Following confirmation of the animals' death, including presence of rigor mortis, lack of heartbeat, respiration and corneal reflex, tumor lesions were excised and photographed. The tumor weight $(\mathrm{g})$, length $(\mathrm{mm})$ and width $(\mathrm{mm})$ was measured. Tumor volume was calculated using the following formula: $\mathrm{Width}^{2} \mathrm{x}$ length/2 (25). The animal study was approved by the Animal Care and Use Committee of The Third Affiliated Hospital of Qiqihar Medical University (Qiqihar, China), complied with Guidelines for the ethical review of laboratory animal welfare People's Republic of China National Standard GB/T 35892-2018.

Luciferase assay. The 3'-untranslated (3'-UTR) regions of Notch1 and Notch2 bearing the putative binding sites of miR-34b-5p were cloned into the psiCHECK-2 plasmid (Promega Corporation). Mutant 3'-UTR luciferase reporter vectors containing 5 mutated nucleotides on the miR-34b-5p binding sites were generated using the QuikChange Multi Site-Directed Mutagenesis kit (Agilent Technologies, Inc.). The plasmids were co-transfected with miR-34b-5p or control miR in RB cells using Lipofectamine 2,000 (Invitrogen; Thermo Fisher Scientific, Inc.). Using the Dual-Luciferase ${ }^{\circledR}$ Reporter (DLR ${ }^{\mathrm{TM}}$ ) Assay System (Promega Corporation), firefly and Renilla luciferase signals were measured at $48 \mathrm{~h}$ post-transfection and the firefly signal was normalized to that of Renilla, as per the manufacturer's instructions. 
Migration and invasion. Cell migration and invasion was measured using Transwell chambers (BD Biosciences) containing 24-well inserts with $8-\mu \mathrm{m}$ pores with or without Matrigel coating (BD Biosciences) according to the manufacturer's protocol. Briefly, The Matrigel matrix aliquot was thawed on ice. Matrigel was mixed with coating buffer thoroughly on ice. A total of $0.1 \mathrm{ml}$ diluted Matrigel matrix coating solution was added to each insert using a sterile pipet. RB cells ( $2 \times 10^{5} /$ well) were seeded in the upper chamber and incubated in RPMI-1640 medium without FBS for 12 and $24 \mathrm{~h}$ for the migration (without Matrigel coating) and invasion (with Matrigel coating) assays at $37^{\circ} \mathrm{C}$ in a $5 \% \mathrm{CO}_{2}$ incubator, respectively. Cells in the upper chamber were then removed, and the remaining cells were fixed in $4 \%$ paraformaldehyde for 5 mins and stained with $0.4 \%$ crystal violet for $1 \mathrm{mins}$ at room temperature. Cells were quantified in five randomly selected fields for each membrane using a light microscope (Nikon Corporation; magnification, $\mathrm{x} 400$ ), and the average cell count of three individual membranes was defined as the migration or invasion index.

TargetScan online tool analysis. The TargetScan online tool (http://www.targetscan.org/vert_72/) was utilized to predict the targets of the miRNAs being assessed.

Statistical analysis. All experiments were performed in triplicate. Data are presented as the mean \pm SD of the three independent sets of experiments. Statistical significance was examined using unpaired Student's t-test or one-way ANOVA followed by Tukey's multiple comparisons test. The correlation between miR-34b-5p and Notch1/Notch2 expression levels was determined using Pearson's correlation analysis. All statistical tests were performed using a licensed copy of SPSS (version 16; SPSS, Inc.). $\mathrm{P}<0.05$ was considered to indicate a statistically significant difference.

\section{Results}

miR-34b-5p is downregulated in tumor tissues and serum of patients with $R B$. The expression level of miR-34b-5p was examined in serum obtained from 10 patients with RB and 10 healthy participants. Additionally, the levels of miR-34b-5p were assessed in tumor tissues and paired adjacent non-tumor tissues. The results indicated that the expression level of miR-34b-5p was significantly downregulated in RB serum (Fig. 1A) in comparison to serum from healthy participants. Moreover, compared with healthy adjacent tissues, miR-34b-5p levels were significantly reduced in RB tissues (Fig. 1B). These results revealed the decreased expression of miR-34b-5p levels in both serum and tumor samples from patients with RB compared with healthy serum and tissues.

miR-34b-5p inhibits $R B$ cell proliferation, migration and invasion. To understand the role of miR-34b-5p in RB, functional assays were performed in RB cell lines, namely Y79 and Weri-Rb-1. The efficiency of transfection was confirmed via $\mathrm{RT}-\mathrm{qPCR}$, which indicated an increase in the expression of miR-34b-5p in RB cells, in comparison to cells transfected with miR controls (Fig. 2A). A comparison of the tumorigenic capacities between the miR-34b-5p and control miR groups was also performed. Cell proliferation was assessed using an MTT assay on Y79 and Weri-Rb-1 cells transfected with miR-34b-5p or control miR. The results indicated that miR-34b-5p transfection significantly reduced RB cell proliferation compared with cells transfected with control miR (Fig. 2B). The proliferative capacity of the cells was also verified using a CCK-8 assay. The results confirmed the findings of the MTT assay, indicating reduced proliferation of Y79 and Wer-Rb-1 cells in the presence of miR-34b-5p (Fig. 2C). In addition, in vivo experiments were performed by subcutaneous transplantation of Y79 cells overexpressing miR-34b-5p which were verified using qRT-PCR (Fig. 2D). Tumor xenografts induced by RB cells overexpressing miR-34b-5p exhibited a slower growth rate and lower weight and volume compared with cell expressing control miR. The largest tumor diameter was $9 \mathrm{~mm}$, and tumor volume was $364.5 \mathrm{~mm}^{3}$ in the control group, while the largest tumor diameter was $3.8 \mathrm{~mm}$, and tumor volume was $27.4 \mathrm{~mm}^{3}$ in the miR-34b-5p group (Fig. 2D). Subsequently, it was investigated whether miR-34b-5p affected RB cell migration and invasion using a Transwell assay. As presented in Fig. S1, miR-34b-5p significantly inhibited RB cell migration and invasion compared with control miR. These results revealed that miR-34b-5p inhibited RB cell proliferation, migration and invasion.

miR-34b-5p reduces $R B$ cell stemness. To assess the effect of miR-34b-5p on RB cell stemness, the expression of CD133, SOX-2 and Nanog, which are sentinel markers of stemness (26), was examined using RT-qPCR. The expression levels of the aforementioned markers were significantly decreased in miR-34b-5p-overexpressing RB cells compared with control cells (Fig. 3A). The alteration in the expression levels was verified via western blotting (Fig. 3B), indicating a change in both mRNA and protein expression. RB stem cell renewal was assessed using a tumor sphere assay. It was observed that the presence of miR-34b-5p significantly reduced RB cell self-renewal ability compared with control miR (Fig. 3C). These results indicated the ability of miR-34b-5p to inhibit stemness in RB cells.

miR-34b-5p directly targets Notch1 and Notch2. Prior to assessing the molecular mechanism that may account for the impact of miR-34b-5p on RB cells, putative targets of miR-34b-5p were predicted using TargetScan online tool. This bioinformatic analysis identified the 3'-UTR of Notch1 and Notch 2 mRNA as potential miR-34b-5p binding targets (Fig. 4A), and further indicated that these sites were conserved. It was hypothesized that Notch1 and Notch2 were potential targets of miR-34b-5p and their dysregulation by miR-34b-5p may serve a role in RB development. This was verified using a luciferase reporter assay involving a luciferase reporter bearing either wild-type or mutant Notch1 or Notch2 3'-UTR. The results indicated that co-transfection with miR-34b-5p significantly repressed luciferase activity compared with control miR (Fig. 4B). Notch1 and Notch2 protein levels were determined in Y79 and Weri-Rb-1 cells following miR-34b-5p transfection. As presented in Fig. 4C, a reduction in the levels of Notch1 and Notch2 expression was observed following transfection with miR-34b-5p in Y79 and Weri-Rb-1 cells, compared with cells transfected with control miR. These 
A

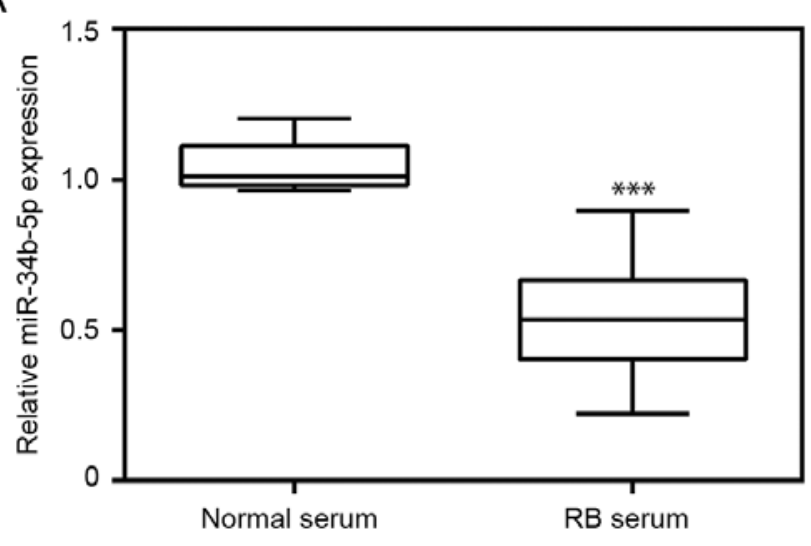

B

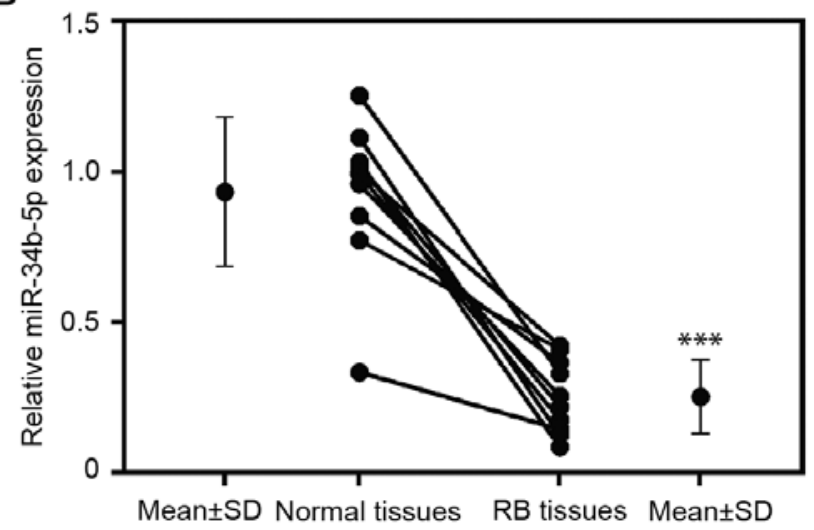

Figure 1. miR-34b-5p expression is downregulated in both the serum and tissues of patients with RB. (A) The expression level of miR-34b-5p was compared in serum samples from 10 patients and 10 healthy participants using reverse transcription-quantitative PCR. (B) miR-34b-5p expression was assessed in RB tissues and paired adjacent healthy tissues excised from patients with $\mathrm{RB} .{ }^{* * *} \mathrm{P}<0.001 \mathrm{vs}$. healthy serum or healthy tissues. miR, microRNA; RB, retinoblastoma.

A

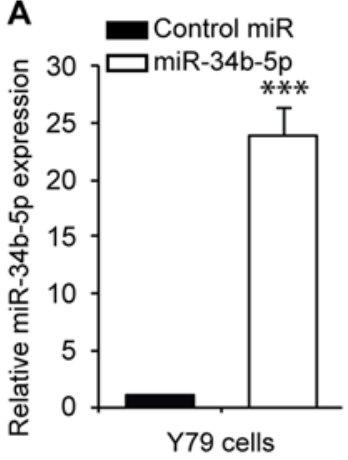

C

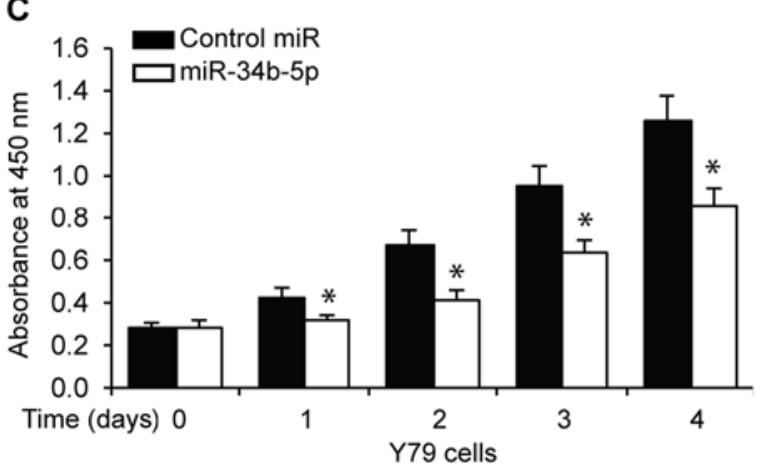

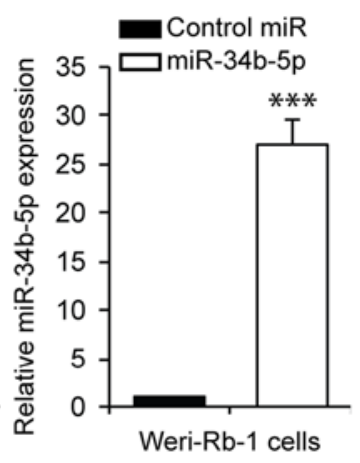

B

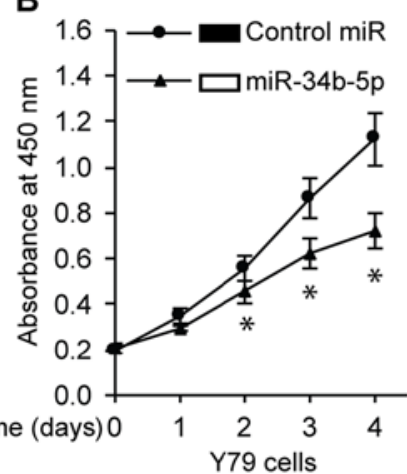

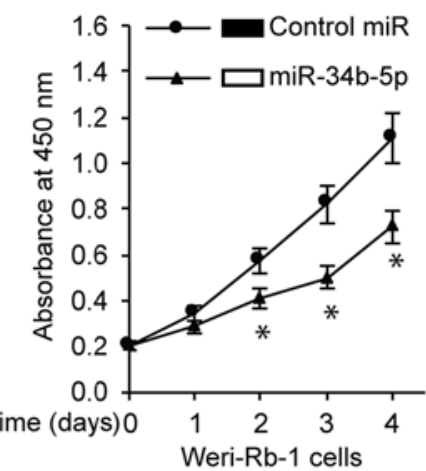

Weri-Rb-1 cells

D
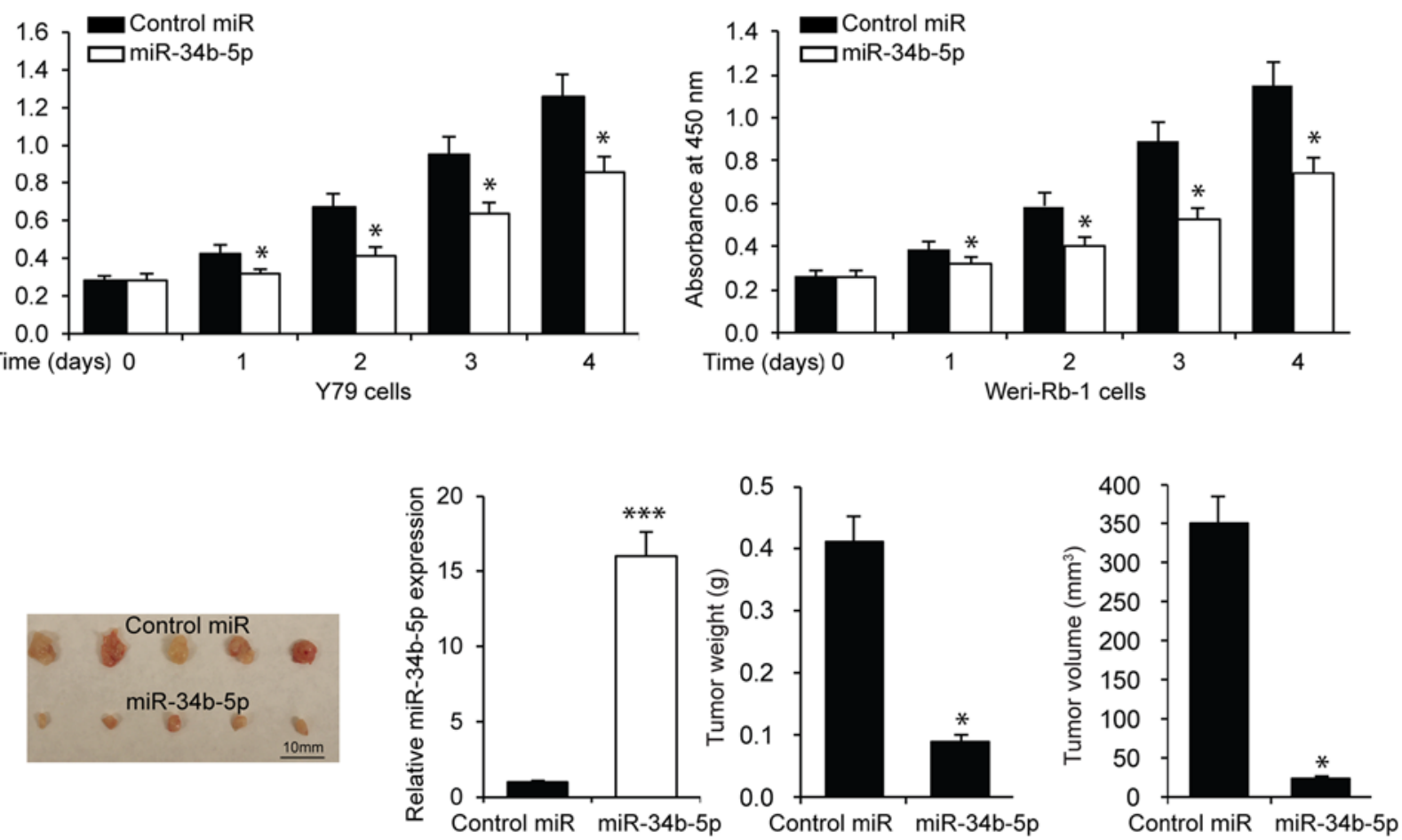

Figure 2. miR-34b-5p reduces RB cell proliferation. (A) miR-34b-5p overexpression was confirmed in Y79 and Weri-Rb-1 cells post-transfection with miR-34b-5p or miR control for $48 \mathrm{~h}$ using reverse transcription-quantitative PCR. (B) MTT assay was performed in Y79 and Weri-Rb-1 RB cells overexpressing miR-34b-5p. (C) Relative cell proliferation was confirmed using Cell Counting Kit-8 assay. (D) Images of tumors and evaluation of tumor weight (g) and volume $\left(\mathrm{mm}^{3}\right)$ after subcutaneous injection of $\mathrm{Y} 79$ cells with miR-34b-5p or control miR for 24 days. Overexpression of miR-34b-5p was verified in the subcutaneous tumor tissues. ${ }^{*} \mathrm{P}<0.05$ and ${ }^{* * * *} \mathrm{P}<0.001$ vs. control miR. miR, microRNA; RB, retinoblastoma. 


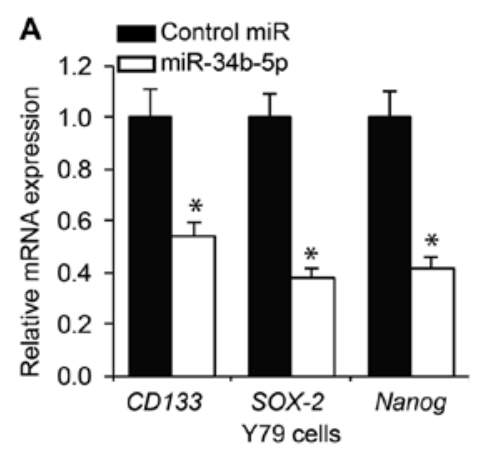

B
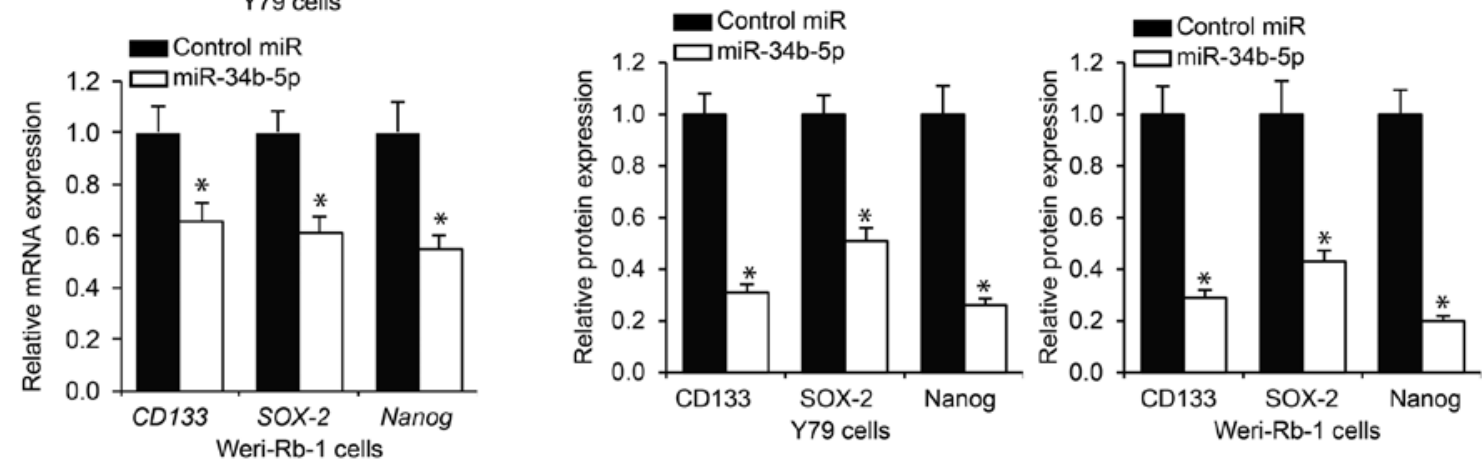

C
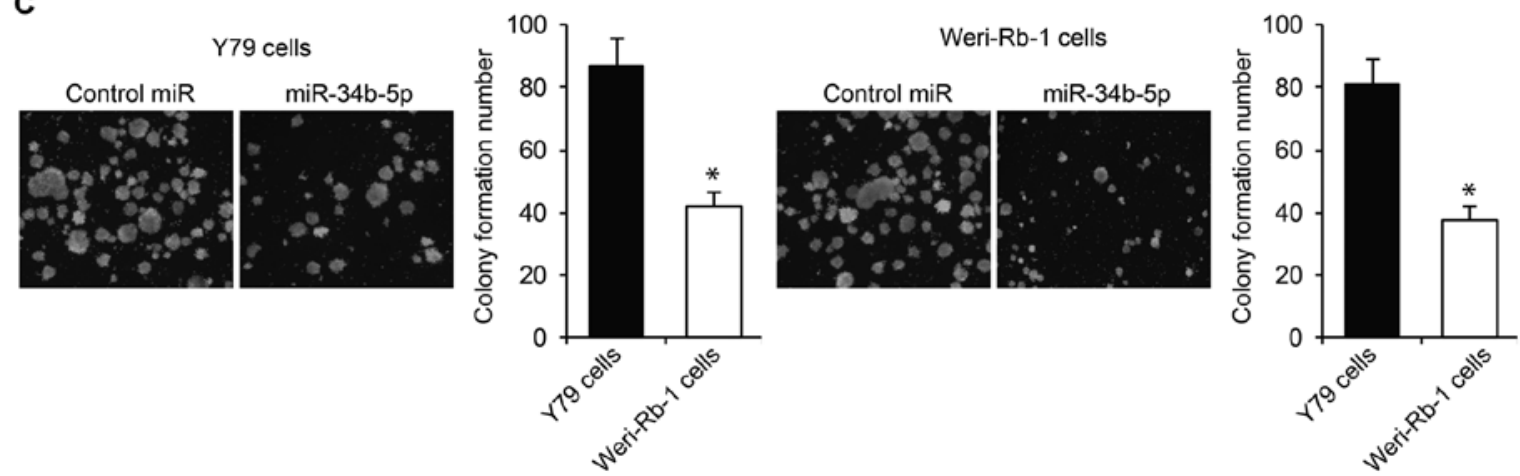

Figure 3. miR-34b-5p reduces RB cell stemness. RB cell stemness in cells transfected with miR-34b-5p was assessed by detecting sentinel stem cell markers, such as CD133, Sox-2 and Nanog, at (A) the transcriptional level via reverse transcription-quantitative PCR and (B) the protein level via western blotting. The density of CD133, Sox-2 and Nanog was quantified relative to $\beta$-actin. (C) Tumor sphere formation was assessed in Y79 and Weri-Rb-1 RB cells transfected with miR-34b-5p (magnification, $\mathrm{x} 200$ ). ${ }^{*} \mathrm{P}<0.05$ vs. control miR. miR, microRNA; RB, retinoblastoma.

observations suggested that Notch1 and Notch2 were possible targets of miR-34b-5p.

Negative correlation between miR-34b-5p and Notch1/2 in $R B$ samples. Pearson's correlation analysis was performed to assess the correlation between miR-34b-5p and its targets, Notch1 and Notch2, in the tumor tissues of patients with RB. A negative correlation was observed between miR-34b-5p and Notch1, and miR-34b-5p and Notch2 expression levels (Fig. 5).

miR-34b-5p inhibits $R B$ cell proliferation and stemness via Notch1 and Notch2. To determine whether miR-34b-5p inhibits RB cell proliferation and stemness via Notch1 and Notch2, rescue experiments were performed by overexpressing Notch 1 or Notch2 in Y79 RB cells transfected with miR-34b-5p. Overexpression of Notch1 or Notch2 was confirmed via western blotting in Y79 RB cells following Notch1 or Notch2 plasmid transfection in comparison to empty vector controls (Fig. S2). Firstly, Notch1 and miR-34b-5p were co-transfected into Y79
RB cells. The overexpression of Notch1 was confirmed in Y79 RB cells co-transfected with miR-34b-5p compared with cells transfected with empty vector (Fig. 6A). These cells were subjected to CCK-8 proliferation and tumor sphere assays. As indicated in Fig. 6B and C, overexpression of Notch1 rescued the decrease in cell proliferation and stemness, which was inhibited by miR-34b-5p. Similarly, overexpression of Notch2 significantly reversed the decrease in RB cell proliferation and stemness induced by miR-34b-5p (Fig. 6D-F).

\section{Discussion}

The present study aimed to assess the role of miR-mediated regulation of Notch1 and Notch2, thereby influencing the initiation and progression of RB. Notch1 was selected as a target gene, owing to its significance in retinal development (27). Additionally, targets beyond $R B 1$, which may or may not be regulating $R B l$, were sought. The association of Notch1 dysregulation with several other malignancies along with 
A

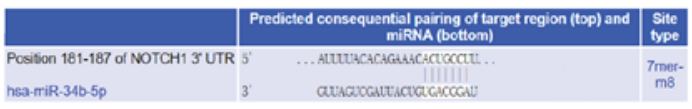

Position 181-187 of Notch1 3' UTR (Mut)

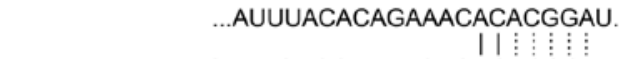

GUUAGUCGAUUACUGUGACGGAU

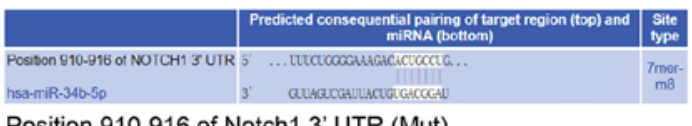

Position 910-916 of Notch1 3' UTR (Mut)

...UUUCUGGGGAAAGACACACGGAG..

GUUAGUCGAUUACUGUGACGG

B
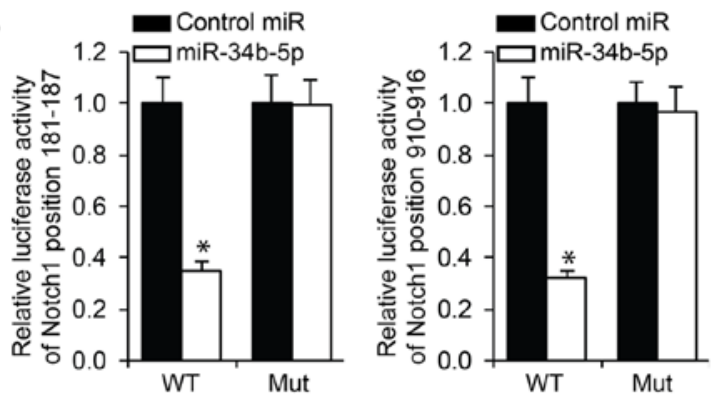

C

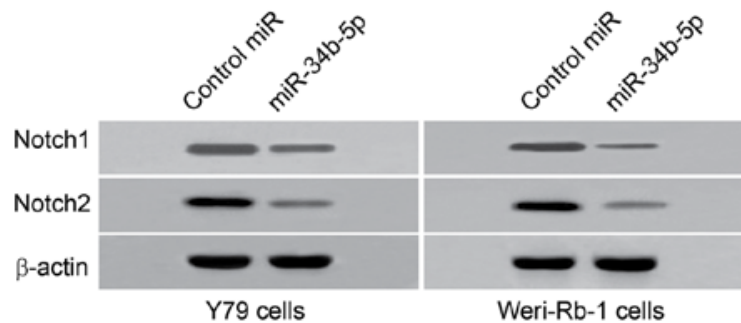

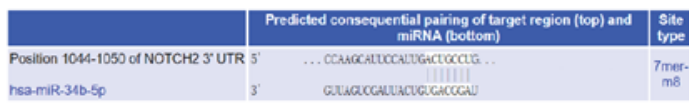

Position 1044-1050 of Notch1 3' UTR (Mut)

..CAAGCAUUCCAUUGACACGGAG

I I $\begin{array}{c:c}\vdots & \vdots \\ \vdots & \vdots\end{array}$

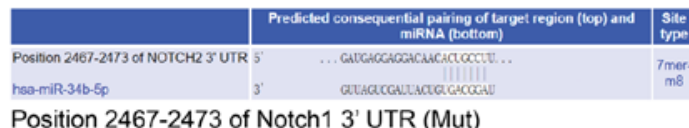

GAUGAGGAGGACAACACACGGAU II $1: \vdots: \vdots: 010$ GUUAGUCGAUUACUGUGACGGAU
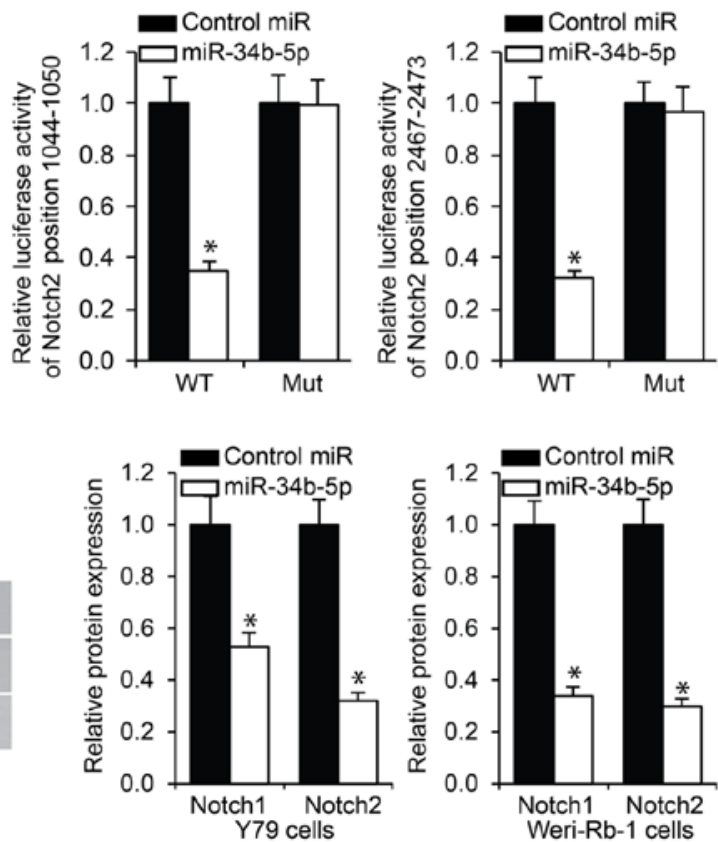

Figure 4. miR-34b-5p directly targets Notch1 and Notch2. (A) Graphical illustration of miR-34b-5p putative binding sites in wild-type Notch1 and Notch2 3'-UTRs and the mutant Notch1 and Notch2 3'-UTRs containing 5 altered nucleotides in the putative target site. (B) Y79 and Weri-Rb-1 retinoblastoma cells transfected with miR-34b-5p or control miR and Notch1 wild-type, Notch1 mutant, Notch2 wild-type or Notch2 mutant 3'-UTR luciferase reporter plasmids were subjected to a luciferase assay. (C) Notch1 and Notch2 expression was detected using western blotting. The density of Notch1 and Notch2 was quantified relative to $\beta$-actin. ${ }^{*} \mathrm{P}<0.05$ vs. control miR. miR, microRNA; UTR, untranslated region; WT, wild type; Mut, mutant.
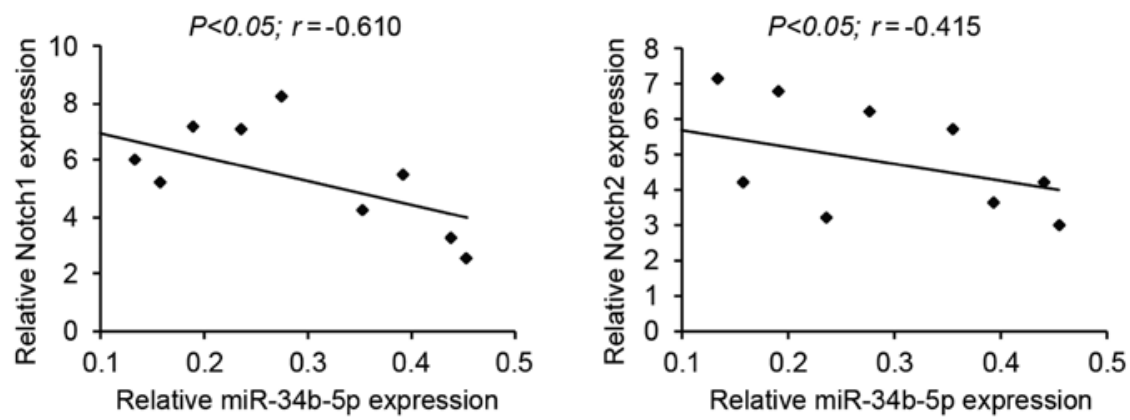

Figure 5. Negative correlation between miR-34b-5p and Notch1 or Notch2 expression levels in RB samples. In 10 tissues from patients with RB, Pearson's correlation analysis was performed between the expression level of miR-34b-5p and that of Notch1 $(\mathrm{P}<0.05, \mathrm{r}=-0.610)$ or $\mathrm{Notch} 2(\mathrm{P}<0.05$, $\mathrm{r}=-0.415)$. miR, microRNA; RB, retinoblastoma.

RB justified the target choice. While the impact of common mutational and non-mutational events on Notch1-induced dysregulation and cancer has been previously studied (20), the present study aimed to elucidate the role of miR-mediated epigenetic modifications in RB. miR-34b-5p as a potential
miR candidate in RB with Notch1 and Notch 2 as its gene targets was identified.

miR-34b-5p is a member of the miR-34 family consisting of miR-34a, miR-34b and miR-34c. It has been previously reported that endogenous expression of miR-34b-5p exhibited 
A
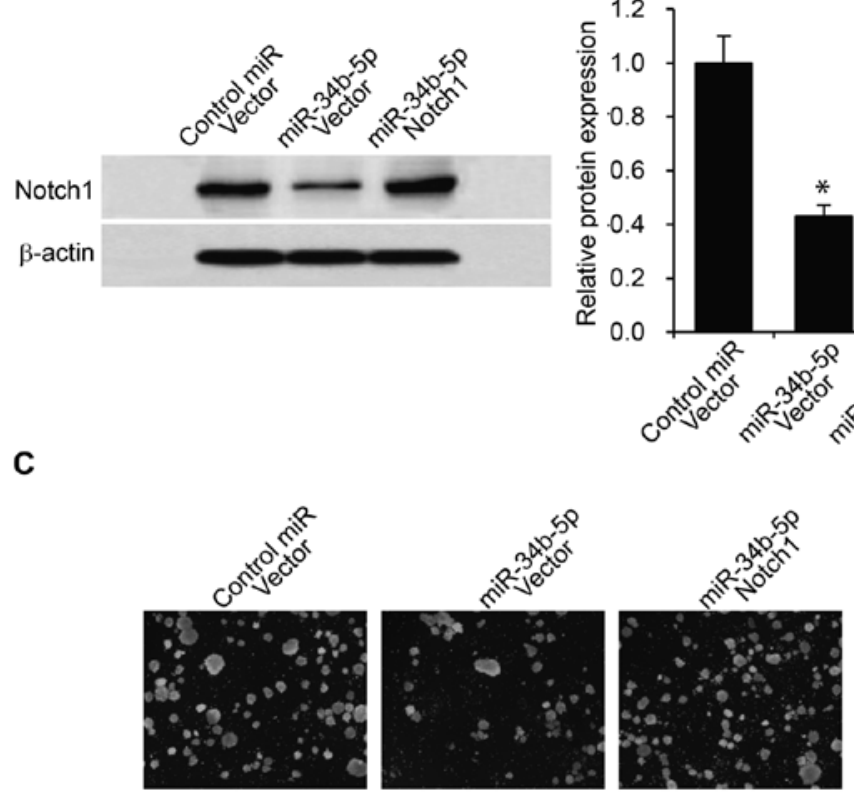

D
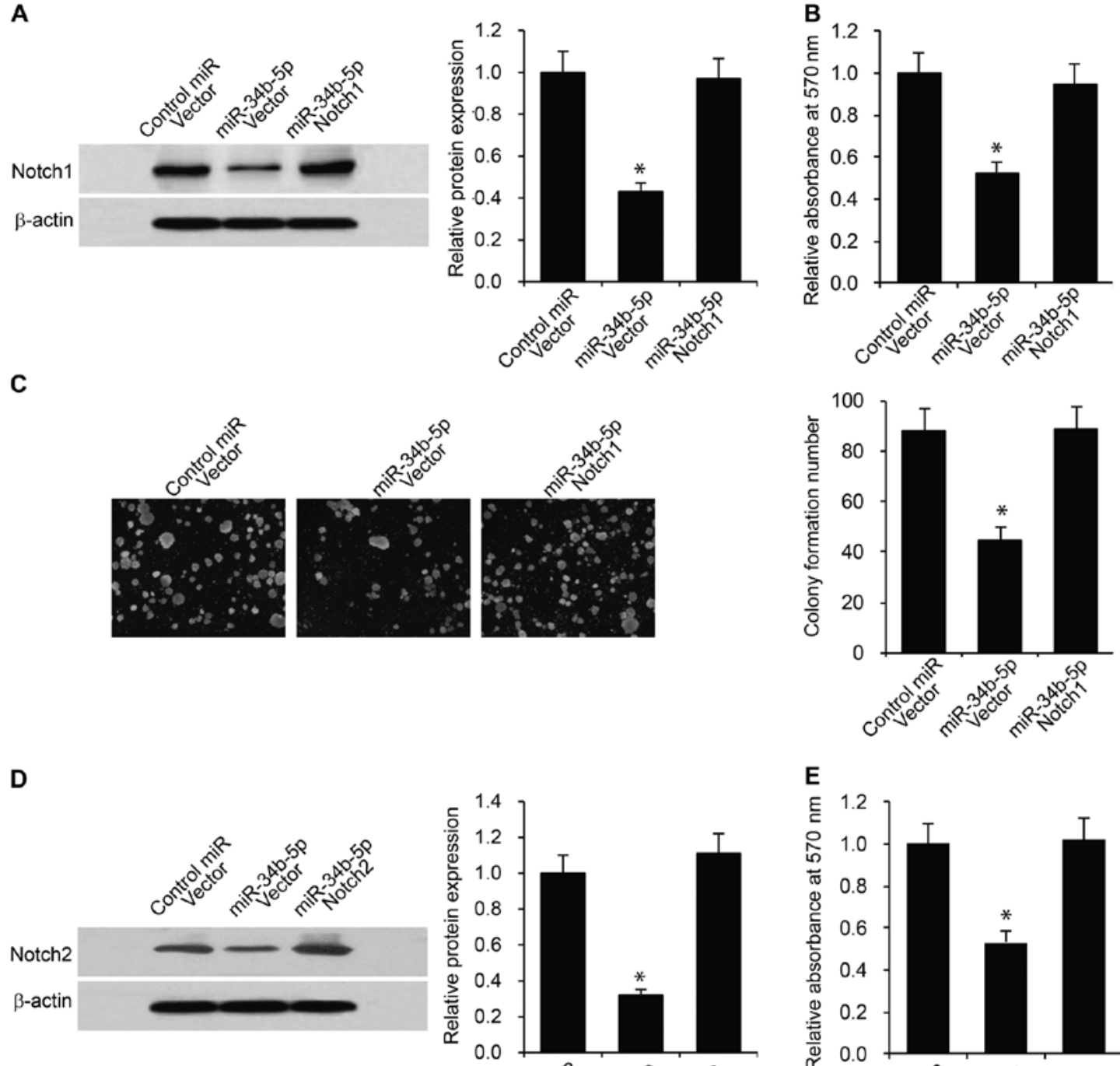

E

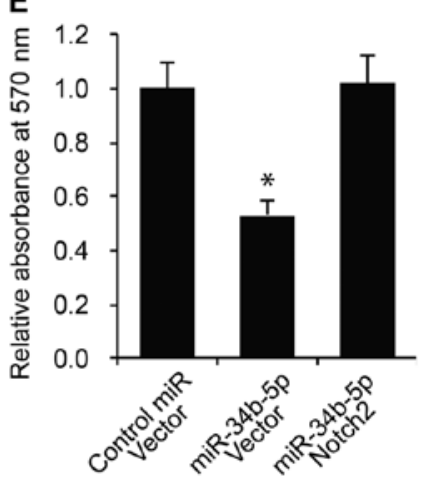

$\mathbf{F}$

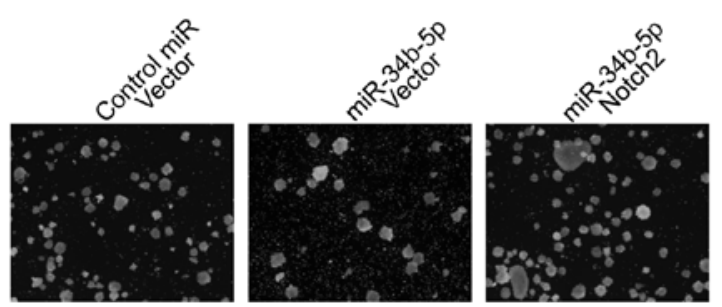

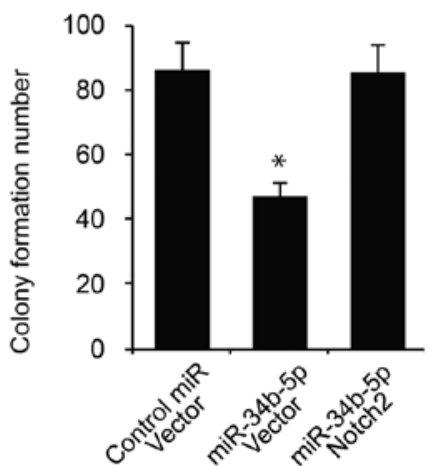

Figure 6. miR-34b-5p inhibits RB cell proliferation and stemness via Notch1 and 2. (A) Western blot analysis of Notch1 expression in Y79 RB cells following transfection with control miR + vector, miR-34b-5p + vector or miR-34b-5p + Notch 1 for $48 \mathrm{~h}$. The density of each group was quantified relative to $\beta$-actin. (B) Relative cell proliferation was determined using CCK-8 assay and (C) a tumor sphere assay was performed in Y79 RB cells following transfection with control miR + vector, miR-34b-5p + vector or miR-34b-5p + Notch1 (magnification, x200). (D) Western blot analysis of Notch2 expression in Y79 RB cells following transfection with control miR + vector, miR-34b-5p + vector or miR-34b-5p + Notch 2 for $48 \mathrm{~h}$. The density of each group was quantified relative to $\beta$-actin. (E) Y79 RB cells co-transfected with control miR + vector, miR-34b-5p + vector or miR-34b-5p + Notch2 were (E) subjected to CCK-8 assay

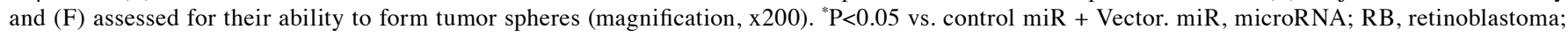
CCK-8, cell counting kit-8.

tumor suppressive characteristics (28). Although the targets of miR-34a are well characterized, the targets of $\mathrm{miR}-34 \mathrm{~b} / \mathrm{c}$ have received little attention, essentially because of the differential expression of the members of the miR-34 family (29). While miR-34a is ubiquitously and highly expressed in the brain tissue, miR-34b and miR-34c are predominantly expressed in 
the lung tissue. Thus, the significance of miR-34b-5p-mediated epigenetic modifications in different cancers has been less explored (28). Furthermore, the correction of an annotation error of miR-34b-5p has resulted in the identification of its function as a tumor suppressor, when endogenously expressed (30). Therefore, while the targets of miR-34a are well characterized (29), the targets of miR-34b are only currently beginning to be explored (31-33). The present study focused on Notch1 and Notch2, which were identified as targets of miR-34b-5p via a bioinformatics-based analysis tool (34), essentially because of the significance of Notch1 and Notch2 in retinal development via the suppression of photoreceptor differentiation and the maintenance of cells in their progenitor states (34). While activation of Notch1 and Notch2 via the upregulation of the ligands Jagged-2 and Delta-like protein 4 is well known, the epigenetic regulation of Notch1 and Notch2 is less studied, to the best of our knowledge (35).

The present study firstly indicated that miR-34b-5p levels were reduced in RB tissues compared with adjacent non-tumor healthy tissues. Moreover, the serum levels of miR-34b-5p were decreased in patient sera as compared with those in age-matched controls. This observation is the first demonstration of dysregulation of miR-34b-5p in RB, to the best of our knowledge.

The overexpression of miR34-b-5p in the RB cell lines Y79 and Weri-Rb-1 was associated with reduced cell growth, expression of sentinel markers of cancer stemness (Nanog, Sox-2 and CD133) and formation of colonies. Additionally, the association of miR-34b-5p with its proposed targets (Notch1 and Notch2) was examined in tissues from patients with RB. The negative correlation that was observed between the levels of miR34b-5p and that of Notch1 or Notch2 suggested an inhibitory role of miR34b-5p in Notch1 and Notch2 expression.

The reduced cell proliferation upon miR34b-5p overexpression in the RB cell lines may be attributed to the role of Notch signaling in $\mathrm{G}_{1} / \mathrm{S}$ progression of the cell cycle, as it has been demonstrated in T cells (36). Canonical and non-canonical Notch signaling pathways may induce the expression of cyclin D3, CDK 4 and CDK 6 (36). Another study in laryngeal squamous cell carcinoma indicated that knockdown of Notch1 was associated with decreased phosphorylation of ERK, AKT, as well as decreased expression of c-Myc, p21, Bcl-2, cyclin D1, CDK4 and Cyclin E, along with increased expression of Bax (37).

The significance of Notch signaling in cancer stem cells (CSC) has been elucidated. Targeting of the Notch signaling pathways via gamma secretase inhibitors (GSIs) has been revealed to decrease cancer cell stemness and is being assessed for its therapeutic benefits (38). In pancreatic cancers, inhibition of Notch activation, via either GSI or Hes-1 short hairpin RNA, resulted in a significant decrease in the proportion of cancer stem cells and tumor sphere formation (38). Hes-1 is a downstream target of Notch1 and influences the maintenance and differentiation of certain stem cells in pancreatic cancer (38). Other mechanisms of Notch-induced CSC include overexpression of the $\mathrm{C}-\mathrm{X}-\mathrm{C}$ chemokine receptor type 4 , which is known to be responsible for stemness-like properties and promotion of chemotaxis via the stromal cell-derived factor 1 axis (12).
In conclusion, the present study demonstrated that the downregulation of miR-34b-5p in RB resulted in the upregulation of Notch1 and Notch2 and was subsequently associated with oncogenic properties, such as dysregulation of cell growth, induction of cancer cell stemness and promotion of tumor sphere formation. The current preliminary investigation suggested a potential therapeutic role for miR-34b-5p in the treatment of RB via regulating the Notch signaling pathway. However, the findings of the present study are preliminary and require further verification via in vivo studies, alongside a more detailed understanding of the regulation of miR-34b-5p in RB.

\section{Acknowledgements}

Not applicable.

\section{Funding}

The present study was supported by the Project of Qiqihar Science and Technology (SFGG-201946)

\section{Availability of data and materials}

The datasets used and/or analyzed during the current study are available from the corresponding author on reasonable request.

\section{Authors' contributions}

SZ and ZC performed experiments, and collected and analyzed the data. SZ wrote the manuscript and ZC designed the experiments. Each author read the manuscript and approved the final version of the manuscript.

\section{Ethics approval and consent to participate}

The current study performed on human samples was approved by the clinical research Ethics Committee of The Third Affiliated Hospital of Qiqihar Medical University (Qiqihar, China) and each patient or healthy subject provided a written informed consent. The animal study was approved by the Animal Care and Use Committee of The Third Affiliated Hospital of Qiqihar Medical University (Qiqihar, China).

\section{Patient consent for publication}

Not applicable.

\section{Competing interests}

The authors declare that they have no competing interests.

\section{References}

1. Dimaras H, Kimani K, Dimba EA, Gronsdahl P, White A, Chan HS and Gallie BL: Retinoblastoma. Lancet 379: 1436-1446, 2012.

2. Fabian ID and Sagoo MS: Understanding retinoblastoma: Epidemiology and genetics. Community Eye Health 31: 7, 2018.

3. Knudson AG Jr, Meadows AT, Nichols WW and Hill R: Chromosomal deletion and retinoblastoma. N Engl J Med 295: 1120-1123, 1976 
4. Chinnam $\mathrm{M}$ and Goodrich DW: RB1, development, and cancer. Curr Top Dev Biol 94: 129-169, 2011.

5. Ishak CA, Coschi CH, Roes MV and Dick FA: Disruption of CDK-resistant chromatin association by pRB causes DNA damage, mitotic errors, and reduces Condensin II recruitment. Cell Cycle 16: 1430-1439, 2017.

6. Mastrangelo D, De Francesco S, Di Leonardo A, Lentini L and Hadjistilianou T: Retinoblastoma epidemiology: Does the evidence matter? Eur J Cancer 43: 1596-1603, 2007.

7. Dimaras H, Khetan V, Halliday W, Orlic M, Prigoda NL, Piovesan B, Marrano P, Corson TW, Eagle RC Jr, Squire JA and Gallie BL: Loss of RB1 induces non-proliferative retinoma: Increasing genomic instability correlates with progression to retinoblastoma. Hum Mol Genet 17: 1363-1372, 2008

8. Dimaras H and Gallie BL: The p75 NTR neurotrophin receptor is a tumor suppressor in human and murine retinoblastoma development. Int J Cancer 122: 2023-2029, 2008.

9. Dimaras H, Coburn B, Pajovic S and Gallie BL: Loss of p75 neurotrophin receptor expression accompanies malignant progression to human and murine retinoblastoma. Mol Carcinog 45: 333-343, 2006.

10. Kandalam M, Mitra M, Subramanian K and Biswas J: Molecular pathology of retinoblastoma. Middle East Afr J Ophthalmol 17: 217-223, 2010

11. Castro-Magdonel BE, Orjuela M, Camacho J, García-Chéquer AJ, Cabrera-Muñoz L, Sadowinski-Pine S, Durán-Figueroa N, Orozco-Romero MJ, Velázquez-Wong AC, Hernández-Ángeles A, et al: miRNome landscape analysis reveals a 30 miRNA core in retinoblastoma. BMC Cancer 17: $458,2017$.

12. Xiao W, Gao Z, Duan Y, Yuan W and Ke Y: Notch signaling plays a crucial role in cancer stem-like cells maintaining stemness and mediating chemotaxis in renal cell carcinoma. J Exp Clin Cancer Res 36: 41, 2017.

13. Xiao W, Chen X and He M: Inhibition of the Jagged/Notch pathway inhibits retinoblastoma cell proliferation via suppressing the PI3K/Akt, Src, p38MAPK and Wnt/ $\beta$-catenin signaling pathways. Mol Med Rep 10: 453-458, 2014

14. Chen CY, Chen YY, Hsieh MS, Ho CC, Chen KY, Shih JY and Yu CJ: Expression of Notch gene and its impact on survival of patients with resectable non-small cell lung cancer. J Cancer 8: 1292-1300, 2017

15. Mollen EWJ, Ient J, Tjan-Heijnen VCG, Boersma LJ, Miele L, Smidt ML and Vooijs MAGG: Moving Breast Cancer Therapy up a Notch. Front Onco 8: 518, 2018.

16. Baker A, Wyatt D, Bocchetta M, Li J, Filipovic A, Green A, Peiffer DS, Fuqua S, Miele L, Albain KS and Osipo C: Notch-1-PTEN-ERK1/2 signaling axis promotes HER2+ breast cancer cell proliferation and stem cell survival. Oncogene 37 4489-4504, 2018.

17. Sun Q, Wang R, Wang Y, Luo J, Wang P and Cheng B: Notch1 is a potential therapeutic target for the treatment of human hepatitis $B$ virus $X$ protein-associated hepatocellular carcinoma. Oncol Rep 31: 933-939, 2014.

18. Venkatesh V, Nataraj R, Thangaraj GS, Karthikeyan M, Gnanasekaran A, Kaginelli SB, Kuppanna G, Kallappa CG and Basalingappa KM: Targeting Notch signalling pathway of cancer stem cells. Stem Cell Investig 5: 5, 2018.

19. Takebe N, Nguyen D and Yang SX: Targeting notch signaling pathway in cancer: Clinical development advances and challenges. Pharmacol Ther 141: 140-149, 2014.

20. Fabbri G, Holmes AB, Viganotti M, Scuoppo C, Belver L, Herranz D, Yan XJ, Kieso Y, Rossi D, Gaidano G, et al: Common nonmutational NOTCH1 activation in chronic lymphocytic leukemia. Proc Natl Acad Sci USA 114: E2911-E2919, 2017.

21. Lee H, Kim KR, Cho NH, Hong SR, Jeong H, Kwon SY, Park KH, An HJ, Kim TH, Kim I, et al: MicroRNA expression profiling and Notch1 and Notch2 expression in minimal deviation adenocarcinoma of uterine cervix. World J Surg Oncol 12: 334, 2014.
22. Maroof H, Islam F, Dong L, Ajjikuttira P, Gopalan V, McMillan NAJ and Lam AK: Liposomal delivery of miR-34b-5p induced cancer cell death in thyroid carcinoma. Cells 7: 265, 2018.

23. Singh $L$ and Kashyap S: Update on pathology of retinoblastoma. Int J Ophthalmol 11: 2011-2016, 2018.

24. Livak KJ and Schmittgen TD: Analysis of relative gene expression data using real-time quantitative PCR and the 2(-Delta Delta C(T)) method. Methods 25: 402-408, 2001

25. Phillips PC, Levow C, Catterall M, Colvin OM, Pastan I and Brem H: Transforming growth factor-alpha-Pseudomonas exotoxin fusion protein (TGF-alpha-PE38) treatment of subcutaneous and intracranial human glioma and medulloblastoma xenografts in athymic mice. Cancer Res 54: 1008-1015, 1994.

26. Zhao D and Cui Z: MicroRNA-361-3p regulates retinoblastoma cell proliferation and stemness by targeting hedgehog signaling. Expe Ther Med 17: 1154-1162, 2019.

27. Jadhav AP, Mason HA and Cepko CL: Notch 1 inhibits photoreceptor production in the developing mammalian retina. Development 133: 913-923, 2006.

28. Zhang L, Liao Y and Tang L: MicroRNA-34 family: A potential tumor suppressor and therapeutic candidate in cancer. J Exp Clin Cancer Res 38: 53, 2019.

29. Misso G, Di Martino MT, De Rosa G, Farooqi AA, Lombardi A, Campani V, Zarone MR, Gullà A, Tagliaferri $\mathrm{P}$, Tassone $\mathrm{P}$ and Caraglia M: Mir-34: A new weapon against cancer? Mol Ther Nucleic Acids 3: e194, 2014.

30. Engkvist ME, Stratford EW, Lorenz S, Meza-Zepeda LA, Myklebost $\mathrm{O}$ and Munthe E: Analysis of the miR-34 family functions in breast cancer reveals annotation error of miR-34b. Sci Rep 7: 9655, 2017.

31. Jiang $L$ and Hermeking H: miR-34a and miR-34b/c suppress intestinal tumorigenesis. Cancer Res 77: 2746-2758, 2017.

32. Majid S, Dar AA, Saini S, Shahryari V, Arora S, Zaman MS, Chang I, Yamamura S, Tanaka Y, Chiyomaru T, et al: miRNA-34b inhibits prostate cancer through demethylation, active chromatin modifications, and AKT pathways. Clin Cancer Res 19: 73-84, 2013.

33. Li Z, Luo Q, Xu H, Zheng M, Abdalla BA, Feng M, Cai B, Zhang X, Nie $Q$ and Zhang X: MiR-34b-5p suppresses melanoma differentiation-associated gene 5 (MDA5) signaling pathway to promote avian leukosis virus subgroup J (ALV-J)-infected cells proliferaction and ALV-J replication. Front Cell Infect Microbiol 7: 17, 2017.

34. Agarwal V, Bell GW, Nam JW and Bartel DP: Predicting effective microRNA target sites in mammalian mRNAs. Elife 4: $\mathrm{e} 05005,2015$

35. Kakuda S, LoPilato RK, Ito A and Haltiwanger RS: Canonical Notch ligands and Fringes have distinct effects on NOTCH1 and NOTCH2. J Biol Chem 295: 14710-14722, 2020.

36. Joshi I, Minter LM, Telfer J, Demarest RM, Capobianco AJ, Aster JC, Sicinski P, Fauq A, Golde TE and Osborne BA: Notch signaling mediates $\mathrm{G} 1 / \mathrm{S}$ cell-cycle progression in $\mathrm{T}$ cells via cyclin D3 and its dependent kinases. Blood 113: 1689-1698, 2009.

37. Dai MY, Fang F, Zou Y, Yi X, Ding YJ, Chen C, Tao ZZ and Chen SM: Downregulation of Notch1 induces apoptosis and inhibits cell proliferation and metastasis in laryngeal squamous cell carcinoma. Oncol Rep 34: 3111-3119, 2015.

38. Abel EV, Kim EJ, Wu J, Hynes M, Bednar F, Proctor E, Wang L, Dziubinski ML and Simeone DM: The Notch pathway is important in maintaining the cancer stem cell population in pancreatic cancer. PLoS One 9: e91983, 2014.

This work is licensed under a Creative Commons Attribution-NonCommercial-NoDerivatives 4.0 International (CC BY-NC-ND 4.0) License. 